\title{
Dynamic Analysis of a Plate on the Generalized Foundation with Fractional Damping Subjected to Random Excitation
}

\author{
Ali Hosseinkhani, ${ }^{1}$ Davood Younesian, ${ }^{1}$ and Saman Farhangdoust $\mathbb{D}^{2}$ \\ ${ }^{1}$ Center of Excellence in Railway Transportation, School of Railway Engineering, Iran University of Science and Technology, \\ Tehran 16846-13114, Iran \\ ${ }^{2}$ Department of Civil and Environmental Engineering, Florida International University, Miami, FL 33174, USA \\ Correspondence should be addressed to Saman Farhangdoust; sfarh006@fiu.edu
}

Received 13 April 2018; Revised 13 June 2018; Accepted 14 June 2018; Published 8 July 2018

Academic Editor: Giovanni Falsone

\begin{abstract}
Copyright (C) 2018 Ali Hosseinkhani et al. This is an open access article distributed under the Creative Commons Attribution License, which permits unrestricted use, distribution, and reproduction in any medium, provided the original work is properly cited.

Stochastic response of a plate on the generalized foundation driven by random excitation is solved in this paper. Governing differential equation is obtained by employing the Galerkin method. The generalized harmonic function technique is applied to the governing equation of motion. Using the stochastic averaging method (SAM), the system is approximated by the time homogeneous diffusive Markov process. Corresponding approximate stationary probability function is achieved by solving associated FokkerPlank-Kolmogorov (FPK). An analytical solution is presented for the stationary probability of the amplitude and velocity. Validity of the stationary probability is verified by Monte-Carlo simulation. Parametric study is carried out to investigate effects of foundation parameters and excitation intensity on the stationary probability function. It is found that the fractional properties act similar to the foundation stiffness and damping and can be employed as a new control parameter for the support design.
\end{abstract}

\section{Introduction}

Fractional calculus has ancient history over 300 years but has been limited to the pure mathematics until new decades. In recent years an increasing interest to the fractional calculus is observed, because of its various applications in physics and engineering $[1,2]$. One of the active branches of fractional calculus is addressed to the material science. Fractional calculus is widely used nowadays in modeling of the viscoelastic fractional material applicable in damping elements in structural and bio applications [3,4].

A plate resting on a viscoelastic foundation can physically model a broad range of engineering problems from runways and slab/panel elements in structural and vehicle engineering to tissue modeling in biomechanics. Free vibration of plates on the generalized nonlinear viscoelastic foundation was analytically studied by Younesian et al. [5]. However, in many real cases, the plate is subject to harmonic or random excitation. In such cases, any kind of geometrical or material nonlinearity in presence of random excitation imposes high complexity to obtain analytical or even numerical solutions. $\mathrm{Xu}$ and
Cheung [6] have extended averaging method to strongly nonlinear systems. Stochastic averaging of a SDOF system under combined harmonic and noise excitation was analyzed by Lal and Singh [7]. Response and stability of a SDOF system subjected to wide-band random excitation were studied by Huang et al. [8]. Zhu et al. introduced a modified stochastic averaging method by use of the energy balance method [9]. Li et al. [10] numerically studied dynamic stability of a column with fractional derivative by use of the averaging method. Spanous et al. presented a standard formulation for modeling vibration of systems with frequency-dependent parameters and fractional properties [11]. They developed a linearizationbased solution to a stochastic nonlinear fractional equation to analyze nonlinear random vibration of a beam with fractional properties [12, 13]. Wahi and Chatterjee studied effect of the delay and fractional derivative terms in oscillations of a resonator [14]. Yang and $\mathrm{Xu}$ used stochastic averaging to investigate the effects of fractional parameters on the output voltage of an energy harvester with fractional damping [15].

In another parallel research line, other researchers have concentrated on the fractional systems under random 
excitations. Huang and Jin [16] employed the stochastic averaging and generalized harmonic functions procedure for a SDOF strongly nonlinear oscillator under Gaussian white noise excitations; they found that the order of fractional derivative has a significant effect on the response of system. Response of a strong nonlinear oscillator [17], its reliability function [18], and its stochastic/asymptotic stability $[19,20]$ have been studied by Chen et al. They could separate fractional derivative into the equivalent quasilinear dissipative force and quasilinear restoring force [21]. Yang et al. studied the stationary and stochastic response of nonlinear system with fractional derivative under white Gaussian noise input $[22,23]$. Failla and Pirrotta presented a numerical method to calculate the response of a system under stochastic excitation based on the discretization of fractional derivative [24].

Noise-induced bifurcation is an undesired phenomenon in stochastic system; In this regard, Sun et al. performed a stochastic sensitivity analysis for the probability response of a nonautonomous system and estimated the critical intensity of noise which leads to explosive bifurcation [25]. Chen et al. derived the amplitude probability density of a Duffing oscillator by the aid of stochastic averaging and by employing the corresponding FPK's equation. Then, they designed a fractional order PID controller in order to eliminate stochastic bifurcations, while the integer-order controller was not able to provide stable response [26]. Chen and Sun presented exact solution for FPK equation of nonlinear systems; they considered an exponential polynomial as the basic function for the response and by an iteration scheme, the basic solution was converged to the exact solution [27]. Yurchenko et al. introduced a suitable change of variable to a vibro-impact system with fractional damping under random excitation; and by use of stochastic averaging, amplitude of mean squared response was presented analytically [28]. Yang et al. obtained an approximate-analytical solution for a noisy vibro-impact system by employing nonsmooth transformation and stochastic averaging and studied stochastic bifurcation phenomena for such systems [29].

In other side, remarkable research has been devoted to identify dynamic characteristics of the conventional plates under random loading. Lal and Singh studied nonlinear free vibration of laminated composite plates resting on elastic foundation with random system properties in thermal environment [7]. Free and forced vibration analysis for the Reissner-Mindlin plates on Pasternak foundation and Winkler elastic foundation were presented by Shen et al. [30]. Dynamic response for a shear deformable plate on two-parameter elastic foundation under transverse dynamic load has been solved by use of perturbation technique by Huang and Zheng [31]. Kostantakopoulos et al. used the mode summation approach and solved dynamic response of a plate on the Winkler foundation under the action of an impact load [32]. Dang-Trung et al. used the Cell-Based Smoothed Triangular Plate Element and analyzed dynamic response of a visco-elastically supported plate under moving load [33]. More recently, Cai et al. used the Galerkin's approach to study dynamic characteristic behavior of a thin plate on fractional Pasternak-type foundation [34]. Reutskiy $[35,36]$ proposed a new methodology based on the combination of the two techniques: the method of external excitation (MEE) and the backward substitution method (BSM) to solve higher order fractional eigenvalue problems. Using statistical linearization-based approach, Malara and Spanos presented large displacement response of a plate with fractional damping nature subjected to random load [37].

According to the reviewed literature, a plate on a foundation can be considered as the mathematical model of several practical problems. In this study, the model of foundation is considered to be the most general form (including fractional, general nonlinearity, and general damping). In such cases, due to mathematical complexity, there are very few reported publications on the context particularly when we have geometrical nonlinearity, fractional properties, and random excitation all together. In many practical applications, properties of the materials used as a foundation or vibration isolator are frequency-dependent. Therefore, conventional models such as Maxwell or Kelvin-Voigt are not accurate in such cases. We in this study show that how fractional characteristics can be applied for this purpose. The objective here is to provide a straightforward methodology to obtain a closed-form solution for a plate resting on one of the most generalized forms of viscoelastic fractional foundation. Presenting the integral-based closed-form solution, in this study, provides the computational platform for design engineers to calculate design parameters of the foundations based on the statistical pattern of the loading condition.

Apart from the methodology, questions about the effects of different material and geometrical properties of the foundation on the probability responses are answered. To this end, the averaging method and harmonic function are employed to solve the governing equation. Then the corresponding Fokker-Planck-Kolmogorov equation is derived and solved to achieve the probability function of the response. The drift and diffusion coefficients are found in a series form for the generalized foundation with fractional damping. MonteCarlo simulation is applied for a plate subjected to a random excitation and validity of the procedure is verified. Effects of the random excitation intensity, the fractional order and foundation parameters on the deflection response probability are examined.

\section{Mathematical Modeling}

The generalized form of nonlinear Duffing stiffness is represented by the following [38]:

$$
g(X)=\sum_{\substack{n=2 k+1 \\ k=1,2, \cdots, m}}^{n} k_{n} X^{n}
$$

where $k_{n}$ includes the linear and nonlinear stiffness coefficients. Corresponding governing equation of a plate motion on the foundation with generalized stiffness and damping is derived as 


$$
\begin{gathered}
D \nabla^{4} w+\rho h \ddot{w}+\varepsilon c_{1} \dot{w}+\varepsilon c_{2} \dot{w}^{3}+\varepsilon a D^{\alpha} w \\
+\sum_{\substack{n=2 k+1 \\
k=1,2, \cdots, m}}^{n} k_{n} w^{n}=\xi(t)
\end{gathered}
$$

where $w=w(x, y, t)$ is the transverse displacement, $D=$ $E h^{3} / 12\left(1-\vartheta^{2}\right)$ denotes the flexural rigidity of the plate and $E, h$ and $\vartheta$ are the Young's modulus, thickness, and Poisson's ratio of the plate, respectively. Also $\nabla^{4}=\left(\partial^{2} / \partial x^{2}+\partial^{2} / \partial y^{2}\right)^{2}$ and $\rho$ is the mass density of the plate material.

At this stage, the Galerkin method is employed to solve the motion equation which suggests the following solution for a simple-supported plate [39]:

$$
w(x, y, t)=\sum_{i=1}^{N} \sum_{j=1}^{M} \sin \left(\frac{i \pi x}{a}\right) \sin \left(\frac{j \pi y}{b}\right) q_{i j}(t)
$$

Substituting (3) into (2) and taking the main mode into account, the following equation is obtained:

$$
\begin{gathered}
\ddot{q}(t)+\beta_{1} \dot{q}(t)+\beta_{2} \dot{q}^{3}(t)+\beta_{3} D^{\alpha} q(t) \\
+\sum_{\substack{n=2 k+1 \\
k=1,2, \ldots, m}}^{n} \lambda_{i} q^{n}(t)=\xi_{1}(t)
\end{gathered}
$$

in which

$$
\begin{aligned}
\beta_{1} & =\frac{\varepsilon c_{1}}{\rho h}, \\
\beta_{2} & =\frac{9 \varepsilon c_{2}}{16 \rho h}, \\
\beta_{3} & =\frac{\varepsilon a}{\rho h}, \\
\xi_{1}(t) & =\frac{16}{\pi^{2}} \xi(t) \\
\lambda_{1} & =\frac{D\left((\pi / a)^{2}+(\pi / b)^{2}\right)^{2}+k_{1}}{\rho h}, \\
\lambda_{i} & =\frac{4 k_{i}}{a b \rho h} \int_{0}^{a} \int_{0}^{b} \sin ^{i+1}\left(\frac{\pi x}{a}\right) \sin ^{i+1}\left(\frac{\pi y}{b}\right) d y d x,
\end{aligned}
$$

\section{Solution Method}

Back to (4), we consider the corresponding nonlinear system with fractional derivative damping subjected to stochastic excitation:

$$
\ddot{X}(t)+\varepsilon h(\dot{X})+\varepsilon a D^{\alpha} X(t)+g(X)=\xi(t)
$$

where

$$
\varepsilon h(\dot{X})=\varepsilon c_{1} \dot{X}(t)+\varepsilon c_{2} \dot{X}^{3}(t)
$$

in which $\varepsilon c_{1}, \varepsilon c_{2}$, and $\varepsilon \alpha$ are the light damping coefficients, $g(X)$ consists of the linear and nonlinear restoring force, and $\xi(t)$ is Gaussian white noise with the following correlation function:

$$
E[\xi(t) \xi(t+\tau)]=2 D_{1} \delta(\tau)
$$

$D^{\alpha}$ donates the fractional derivative operator and according to Riemann-Liouville's definition is represented by the following $[40,41]$ :

$$
D^{\alpha} X(t)=\frac{1}{\Gamma(1-\alpha)} \frac{d}{d t} \int_{0}^{t} \frac{X(t-\tau)}{\tau^{\alpha}} d \tau, \quad 0<\alpha<1
$$

The most well-known, practical representations for fractional derivative operators are Riemann-Liouville (RL), Caputo, and Grunwald-Letnikov (GL) definitions. For many cases, it has been proved that these fractional derivative definitions are equivalent. Grunwald-Letnikov definition presents the relation in the form of a series and is more appropriate for discrete systems. Riemann-Liouville definition is widely used for calculations of analytical solutions in continues systems. Caputo definition can include the initial conditions of a problem in the same form of nonfractional problems; so it can provide a physical meaning for the initial conditions. In the present study, according to the nature of stochastic analysis and continuous modeling hypothesis, definition of RiemannLiouville is taken into account.

The free conservative vibration associated with the system (6) is therefore represented by the following:

$$
\ddot{X}(t)+g(X)=0
$$

The response of system (6) is a nearly random spread of the system (10): therefore, by use of generalized harmonic function, the following form of response is assumed for the following solution:

$$
\begin{aligned}
X(t) & =A \cos \theta(t) \\
\dot{X}(t) & =-A v \sin \theta(t) \\
\theta(t) & =\Phi(t)+\Gamma(t) \\
v(A, \theta) & =\frac{d \Phi}{d t}=\sqrt{\frac{2(U(A)-U(A \cos \theta))}{A^{2} \sin ^{2} \theta}}
\end{aligned}
$$

$A, \Phi$, and $\Gamma$ are the random process functions. By applying the generalized Van der Pol transformation from $X, \dot{X}$ to $A$ and $\Gamma$, (11a) and (11b) yield to

$$
\begin{aligned}
\frac{d A}{d t} & =\varepsilon F_{1}+G_{1} \xi(t) \\
\frac{d \Gamma}{d t} & =\varepsilon F_{2}+G_{2} \xi(t)
\end{aligned}
$$


in which

$$
\begin{aligned}
F_{1} & =F_{11}+F_{12} \\
F_{11} & =\frac{A v \sin \theta}{g(A)} a D^{\alpha}(A \cos \theta) \\
F_{12} & =\frac{A v \sin \theta}{g(A)} h(-A v \sin \theta) \\
F_{2} & =\frac{v \cos \theta}{g(A)} a D^{\alpha}(A \cos \theta)+\frac{v \cos \theta}{g(A)} h(-A v \sin \theta) \\
G_{1} & =\frac{-A v \sin \theta}{g(A)} \\
G_{2} & =\frac{-v \cos \theta}{g(A)}
\end{aligned}
$$

Since (12a) is independent from $\Gamma$, the Itô equation related to $A$ should be satisfied:

$$
d A=m(A) d t+\sigma(A) d B(t)
$$

where $m(A)$ and $\sigma(A)$ are the draft and diffusion coefficients:

$$
\begin{aligned}
m(A) & =\varepsilon\left\langle F_{11}+F_{12}+D_{1} \frac{d G_{1}}{d A} G_{1}+D_{1} \frac{d G_{1}}{d \Gamma} G_{2}\right\rangle_{\theta} \\
\sigma^{2}(A) & =\varepsilon D_{1}\left\langle 2 G_{1}^{2}\right\rangle_{\theta}
\end{aligned}
$$

in which $\langle.\rangle_{\theta}$ represents the average operator with respect to $\theta$ :

$$
\langle.\rangle_{\theta}=\lim _{T \longrightarrow \infty} \frac{1}{T} \int_{0}^{T}\langle.\rangle d t=\frac{1}{2 \pi} \int_{0}^{2 \pi}\langle.\rangle d \theta
$$

Since $A$ and $\Gamma$ are slowly varying with time, $\theta(t)$ could be approximated by the instantaneous frequency:

$$
\theta(t-\tau) \approx \theta(t)-w(A) \tau
$$

By use of (17) and averaging over the first term of (15), $\left\langle F_{11}\right\rangle_{\theta}$ is reduced to

$$
\begin{aligned}
\left\langle F_{11}\right\rangle_{\theta}=\frac{a}{g(A)} \lim _{T \rightarrow \infty} \frac{1}{T} \int_{0}^{T} D^{\alpha}(A \cos \theta) \times A v \sin \theta d t \\
=\frac{a}{g(A) \Gamma(1-\alpha)} \lim _{T \rightarrow \infty} \frac{1}{T} \\
\quad \cdot \int_{0}^{T} A v \sin \theta d\left(\int_{0}^{t} \frac{A \cos \theta(t-\tau)}{\tau^{\alpha}} d \tau\right) \\
=\frac{a}{g(A) \Gamma(1-\alpha)} \\
\quad \lim _{T \rightarrow \infty}\left\{\left.\frac{1}{T}\left(A v \sin \theta \int_{0}^{t} \frac{A \cos \theta(t-\tau)}{\tau^{\alpha}} d \tau\right)\right|_{0} ^{T}\right. \\
\left.\quad-\frac{1}{T} \int_{0}^{T}\left(\int_{0}^{t} \frac{\cos \theta(t-\tau)}{\tau^{\alpha}} d \tau\right) \frac{d}{d t}(A v \sin \theta) d t\right\}
\end{aligned}
$$

Using the following asymptotic integrals

$$
\begin{aligned}
& \int_{0}^{t} \frac{\cos (\omega \tau)}{\tau^{q}} d \tau=\omega^{(q-1)} \int_{0}^{s} \frac{\cos (u)}{u^{q}} d u \\
& =\omega^{(q-1)}\left(\Gamma(1-q) \sin \left(\frac{q \pi}{2}\right)+\frac{\sin (s)}{s^{q}}+O\left(s^{-q-1}\right)\right) \\
& \int_{0}^{t} \frac{\sin (\omega \tau)}{\tau^{q}} d \tau=\omega^{(q-1)} \int_{0}^{s} \frac{\sin (u)}{u^{q}} d u \\
& =\omega^{(q-1)}\left(\Gamma(1-q) \cos \left(\frac{q \pi}{2}\right)-\frac{\cos (s)}{s^{q}}+O\left(s^{-q-1}\right)\right)
\end{aligned}
$$

We can simplify (18) to

$$
\begin{aligned}
& \left\langle F_{11}\right\rangle_{\theta} \approx \frac{-a}{g(A) \Gamma(1-\alpha)} \lim _{T \rightarrow \infty} \frac{1}{T} \int_{0}^{T} A g(A \cos \theta) \\
& \cdot\left[\cos \theta \int_{0}^{t} \frac{\cos (\omega(A) \tau)}{\tau^{q}} d \tau\right. \\
& \left.\quad+\sin \theta \int_{0}^{t} \frac{\sin (\omega(A) \tau)}{\tau^{q}} d \tau\right] \\
& \approx \frac{-A a}{2 \pi g(A) \omega^{1-\alpha}} \int_{0}^{2 \pi} g(A \cos \theta)\left[\cos \theta \sin \left(\frac{\alpha \pi}{2}\right)\right. \\
& \left.\quad+\sin \theta \cos \left(\frac{\alpha \pi}{2}\right)\right] d \theta
\end{aligned}
$$

In the following step, $G_{i}$ is expanded to the Fourier series with respect to $\theta$ :

$$
\begin{array}{r}
G_{i}=G_{i 0}(A)+\sum_{n=1}^{\infty} G_{i n}^{(c)}(A) \cos n \theta+G_{\text {in }}^{(s)}(A) \sin n \theta, \\
i=1,2
\end{array}
$$

By substituting $G_{i}$ and $F_{11}$ into (13) the averaged drift and diffusion coefficients are obtained as

$$
\begin{gathered}
\bar{m}(A)=\varepsilon\left\langle F_{11}\right\rangle_{\theta}+\frac{\varepsilon A}{g(A)} \int_{0}^{2 \pi} v \sin \theta h(-A v \sin \theta) d \theta \\
+\varepsilon D_{1}\left[\frac{d G_{10}}{d A} G_{10}+\frac{1}{2} \sum_{n=1}^{\infty} \frac{d G_{1 n}^{(c)}}{d A} G_{1 n}^{(c)}+\frac{d G_{1 n}^{(s)}}{d A} G_{1 n}^{(s)}\right. \\
\left.+n G_{1 n}^{(s)} G_{2 n}^{(c)}-n G_{1 n}^{(c)} G_{2 n}^{(s)}\right] \\
\bar{\sigma}^{2}(A)=\varepsilon D_{1}\left[2 G_{10}^{2}+\sum_{n=1}^{\infty} G_{1 n}^{(c)}+G_{1 n}^{(s)}\right]
\end{gathered}
$$

The FPK's equation associated with (14) is therefore derived as

$$
\frac{\partial p}{\partial t}=-\frac{\partial}{\partial A}[\bar{m}(A) p]+\frac{1}{2} \frac{\partial^{2}}{\partial A^{2}}\left[\bar{\sigma}^{2}(A) p\right]
$$


Since $g(X)$ is a monotonic increasing function, the boundary condition for (23) should satisfy

$$
\begin{aligned}
& \text { if }: A=0 \Longrightarrow p=\text { finite } \\
& \text { if }: A \longrightarrow \infty \Longrightarrow p, \frac{\partial p}{\partial A} \longrightarrow 0
\end{aligned}
$$

Therefore, the stationary response of FPK's equation is

$$
p(A)=\frac{C}{\sigma^{2}(A)} \exp \left[\int_{0}^{A} \frac{2 m(u)}{\sigma^{2}(u)} d u\right]
$$

where $C$ is the normalization constant. Back to the main problem, corresponding potential energy for the generalized restoring force is given by

$$
U(X)=\int_{0}^{X} g(x) d x=\sum_{\substack{n=2 k+1 \\ k=1,2, \cdots, m}}^{n} \frac{k_{n} X^{n+1}}{n+1}
$$

At this step, drift and diffusion coefficients can be extracted for the original system with (4). Using (1), the integral of (20) can be expressed as

$$
\left\langle F_{11}\right\rangle_{\theta}=-\frac{A \beta_{3} \sum_{\substack{n=2 k+1 \\ k=1,2, \ldots, m}}^{n}\left(\lambda_{i} A^{n} \int_{0}^{2 \pi} \cos ^{n+1} u d u\right)}{2 \pi \lambda_{1}^{(1-\alpha) / 2} \sum_{\substack{n=2 k+1 \\ k=1,2, \ldots, m}}^{n} \lambda_{i} A^{n}}
$$

where $\lambda_{1}=\omega^{2}$. Using definition of h, i.e., (7) with the coefficients defined by (5), we have

$$
\begin{aligned}
& \frac{A}{g(A)} \int_{0}^{2 \pi} v \sin \theta h(-A v \sin \theta) d \theta \\
& \quad=\frac{-A^{2} \sum_{\substack{n=2 k+1 \\
k=1,2, \ldots, m}}^{n}\left\{\beta_{1} \int_{0}^{2 \pi}\left(2 \lambda_{n} /(n+1)\right)\left(1-\cos ^{n+1} u\right) d u+\beta_{2}\left[\int_{0}^{2 \pi}\left(2 \lambda_{n} /(n+1)\right)\left(1-\cos ^{n+1} u\right) d u\right]^{2}\right\}}{2 \pi \sum_{\substack{n=2 k+1 \\
k=1,2, \ldots, m}}^{n} \lambda_{i} A^{n}}
\end{aligned}
$$

By substituting (27), (28) into (22), the drift and diffusion coefficients for the main equation are consequently obtained as $(\varepsilon=1)$ :

$$
\begin{aligned}
m(A)= & \frac{-A^{2} \sum_{\substack{n=2 k+1 \\
k=1,2, \ldots, m}}^{n}\left\{\beta_{1} \int_{0}^{2 \pi}\left(2 \lambda_{n} /(n+1)\right)\left(1-\cos ^{n+1} u\right) d u+\beta_{2}\left[\int_{0}^{2 \pi}\left(2 \lambda_{n} /(n+1)\right)\left(1-\cos ^{n+1} u\right) d u\right]^{2}\right\}}{2 \pi \sum_{\substack{n=2 k+1 \\
k=1,2, \ldots, m}}^{n} \lambda_{i} A^{n}} \\
& -\frac{A \beta_{3} \sum_{\substack{n=2 k+1 \\
k=1,2, \ldots, m}}^{n}\left(\lambda_{i} A^{n} \int_{0}^{2 \pi} \cos ^{n+1} u d u\right)}{2 \pi \lambda_{1}^{(1-\alpha) / 2} \sum_{\substack{n=2 k+1 \\
k=1,2, \ldots, m}}^{n} \lambda_{i} A^{n}}+\frac{D_{1}}{2}\left[\frac{d G_{11}^{(s)}}{d A} G_{11}^{(s)}+G_{11}^{(s)} G_{21}^{(c)}\right] \\
\sigma^{2}(A)= & D_{1}\left[G_{11}^{(s)}\right]^{2}
\end{aligned}
$$

where $G_{i 1}$ is obtained according to (21):

$$
\begin{aligned}
& G_{11}^{(s)}=\frac{-A\left(b_{0}-b_{2} / 2\right)}{\sum_{\substack{n=2 k+1 \\
k=1,2, \ldots, m}}^{n} \lambda_{i} A^{n}}, \\
& G_{21}^{(c)}=\frac{-\left(b_{0}+b_{2} / 2\right)}{\sum_{\substack{n=2 k+1 \\
k=1,2, \ldots, m}}^{n} \lambda_{i} A^{n}}
\end{aligned}
$$

$b_{0}$ and $b_{2}$ are calculated from the following approximation:

$$
v(A, \theta)=b_{0}+b_{2} \cos 2 \theta+b_{4} \cos 4 \theta+b_{6} \cos 6 \theta
$$

Using (11b) and (26), we have the following relation for $v(A, \theta)$ :

$$
v(A, \theta)=(\eta(1+u))^{0.5}
$$

where

$$
\eta=\sum_{\substack{n=2 k+1 \\ k=1,2, \cdots m}}^{n} \frac{2 \lambda_{n} A^{n-1}}{n+1}
$$

$$
u=\sum_{\substack{n=2 k+1 \\ k=1,2, \cdots m}}^{n} \eta_{n} \cos ^{n-1} \theta
$$




$$
\eta_{j}=\frac{1}{\eta} \sum_{i=j}^{n-1} \frac{2 \lambda_{i} A^{i-1}}{i+1}, \quad j=3,5, \ldots, n
$$

$b_{0}$ and $b_{2}$ are then obtained from (31) and (33):

$$
\begin{aligned}
& b_{0}=\frac{\sqrt{\eta}}{2 \pi} \int_{0}^{2 \pi} \sqrt{1-u} d \theta \\
& b_{0}=\frac{\sqrt{\eta}}{\pi} \int_{0}^{2 \pi} \sqrt{1-u} \cos 2 \theta d \theta
\end{aligned}
$$

$$
\sqrt{1-u} \approx 1+\frac{u}{2}-\frac{u^{2}}{8}+\frac{u^{3}}{16}
$$

The stationary probability density function is obtained by substituting $A=U^{-1}(H)$ in the following relation:

$$
p(H)=p(A)\left|\frac{d A}{d H}\right|=\left.\frac{p(A)}{g(A)}\right|_{A=U^{-1}(H)}
$$

in which $U^{-1}$ is the inverse of potential function. The joint stationary probability of displacement and velocity can also be obtained as

$$
p\left(x_{1}, x_{2}\right)=\left.\frac{p(H)}{T(H)}\right|_{H=x_{2}^{2}+U\left(x_{1}\right)}
$$

in which

$$
T(H)=\left.\frac{2 \pi}{w(H)}\right|_{H=U^{-1}(A)}
$$

Finally, the stationary probability density function of displacement and velocity are obtained to be

$$
\begin{aligned}
& p\left(x_{1}\right)=\int_{-\infty}^{\infty} p\left(x_{1}, x_{2}\right) d x_{2} \\
& p\left(x_{2}\right)=\int_{-\infty}^{\infty} p\left(x_{1}, x_{2}\right) d x_{1}
\end{aligned}
$$

and the stationary mean amplitude can be obtained by

$$
E[A]=\int_{0}^{\infty} A p(A) d A
$$

\section{Numerical Results}

In this section, numerical results are presented and discussed for a case study. The aim is to show validity of the solution procedure and to provide a parametric study. The validation is performed by the comparison between the results from the present formulation and the results from Monte-Carlo simulation. Monte-Carlo simulation takes all of the parameters with uncertainty into account and presents the probability distribution for a parameter by performing the calculation over and over. By taking all of the calculated results for a desired parameter into account, the corresponding probability density or probability distribution can be achieved.

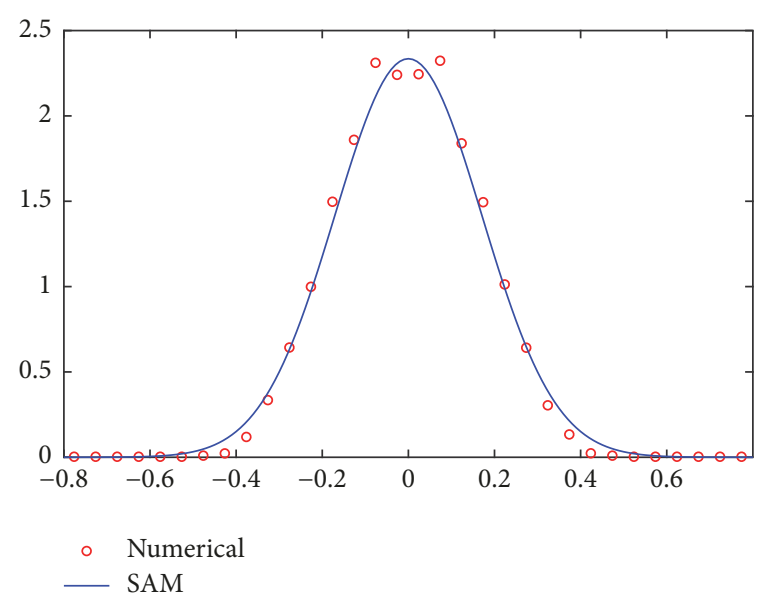

FIGURE 1: The stationary probability density of the displacement.

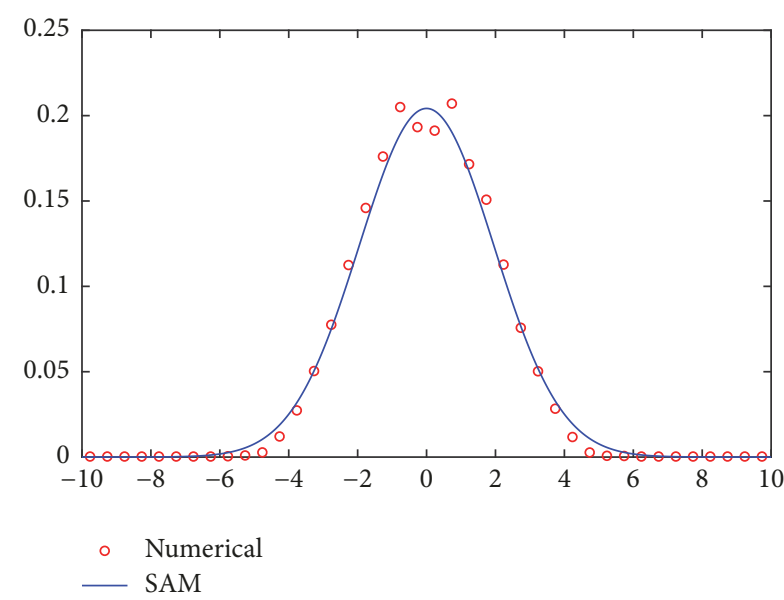

FIGURE 2: The stationary probability density of the velocity.

Parameters of the plate are set as: $\mathrm{a}=4 \mathrm{~m}, \mathrm{~b}=2 \mathrm{~m}, \mathrm{~h}$ $=2 \mathrm{~mm}, \mathrm{E}=207 \mathrm{Gpa}, \vartheta=0.3, \rho=7800 \mathrm{~kg} / \mathrm{m}^{3}$. Properties of the foundation are $c_{1}=0.4 \mathrm{Ns} / \mathrm{m}, c_{2}=0, a=1$ $N s^{\alpha} / m, \alpha=0.55, D_{1}=0.1, k_{1}=600 \mathrm{~N} / \mathrm{m}, k_{3}=12$ $\mathrm{kN} / \mathrm{m}^{3}, k_{5}=25 \mathrm{kN} / \mathrm{m}^{5}, k_{7}=40 \mathrm{kN} / \mathrm{m}^{7}, k_{9}=k_{11}=\ldots=0$. Drift and diffusion coefficients are obtained for such a system according to the previous section. Stationary probability and expected value can be calculated from (38) and (39). Figures 1 and 2 show the stationary probability density function of amplitude and velocity, respectively. The solid lines are correlated with the SAM and dots are outcomes of the MonteCarlo numerical simulation obtained from Figures 3 and 4.

The displacement response and velocity response samples from Monte-Carlo numerical simulation are illustrated in Figures 3 and 4 . The joint probability density function between the deflection and velocity is illustrated in Figure 5. A good agreement between the analytical proposed solution and those from numerical simulation is observed in these figures.

In the following parts, effects of the foundation properties on the stationary responses are investigated. Figure 6 illustrates influence of the power order of the stiffness on 


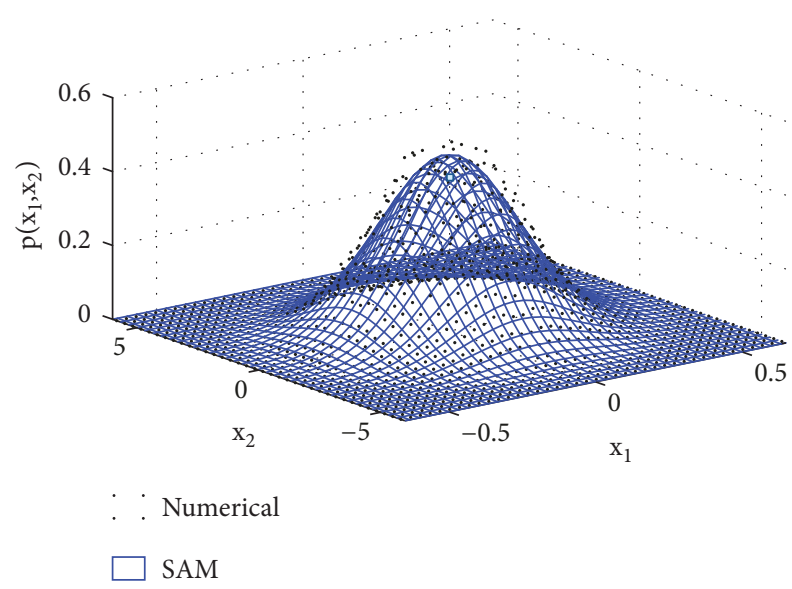

Figure 3: The joint stationary probability density between the displacement and velocity.

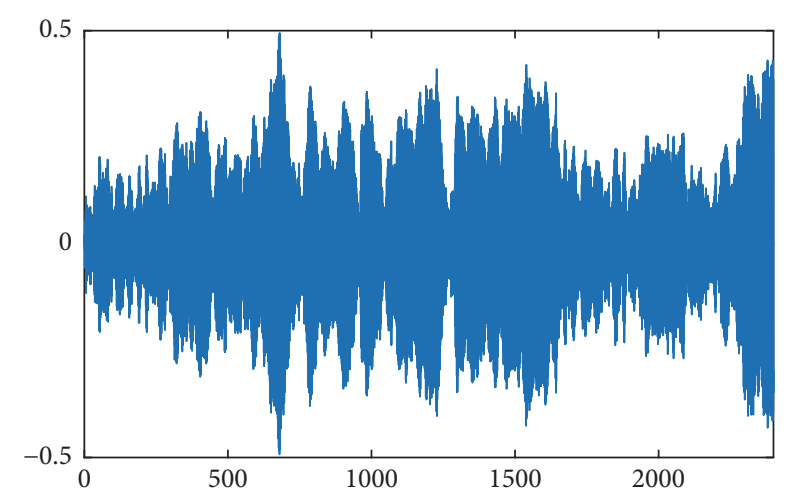

Figure 4: Displacement response from Monte-Carlo simulation.

the stationary probability amplitude. It is seen that for larger powers we get more concentrated probability function around a smaller value of amplitude.

The effect of fractional order derivative on stationary probability amplitude is illustrated in Figure 7. It can be seen that the fractional derivative order has a remarkable effect on the responses. It acts similar to the power order of the stiffness; i.e., larger fractional order gives a more condensed probability function centered in smaller amplitudes.

Figure 8 shows variation of the amplitude probability function for four different values of fractional damping coefficient. It is observed that having smaller fractional damping coefficient gives a broadly spread probability. It is also seen that the sensitivity of the system is quite higher with respect to this parameter at large values of fractional damping. Figure 9 illustrates the effect of nonlinear damping coefficient $c_{2}$ on the stationary probability density of the amplitude. It is seen that sensitivity of the responses with respect $c_{2}$ decreases at high values of the nonlinear damping.

Variation of the expected value versus the fractional order derivative is illustrated for $n=1$ and $n=3$ in Figure 10. It is seen that the expected value decreases with increasing $\alpha$ and its decreasing rate enhances as $\alpha$ increases. It is also seen that the sensitivity with respect to $\alpha$ is higher when $n=1$.

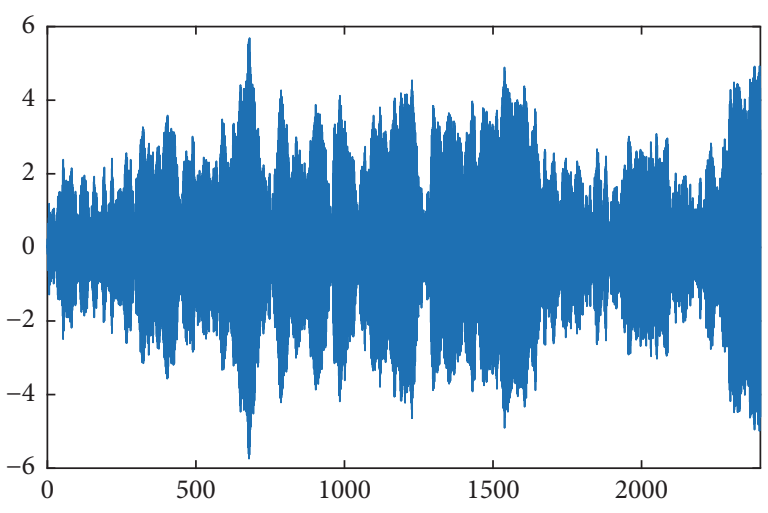

Figure 5: Velocity response from Monte-Carlo simulation.

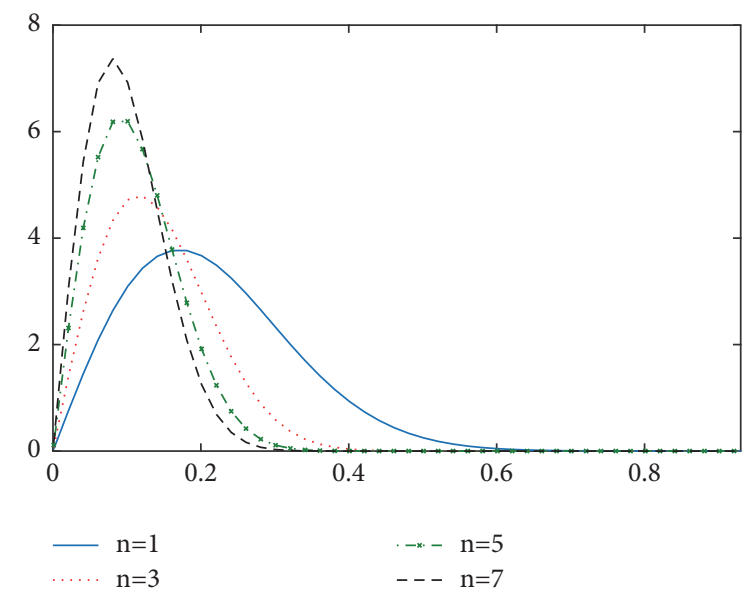

FIgURE 6: Effect of the power order of stiffness on the probability function of amplitude.

\section{Conclusions}

A straightforward and closed-form solution was proposed for nonlinear vibration analysis of a supported plate subjected to stochastic excitation. The support was modeled by a generalized form of stiffness and damping with fractional properties. Generalized harmonic function in conjunction with the stochastic averaging method was used to extract the averaged Itô and FPK's equation. Validity of the solution procedure was numerically verified by use of Monte-Carlo simulation. It was shown that the stationary probability density function of the responses is remarkably influenced by the fractional parameters. This means that instead of changing the stiffness and damping of the foundation which is costly and hard to implement, designers can use a fractional layer. Similar to the order of foundation stiffness, it was found that larger fractional order corresponds to more condensed probability function and to smaller amplitudes. It was proved that the fractional damping coefficient acts similar to the nonlinear damping but with an extra benefit. Having more sensitivity at high fractional damping reduces the risk of saturation at high material damping. It was also shown that for a linear foundation we can have desirable sensitivity with respect to fractional order so that instead of adding extra 


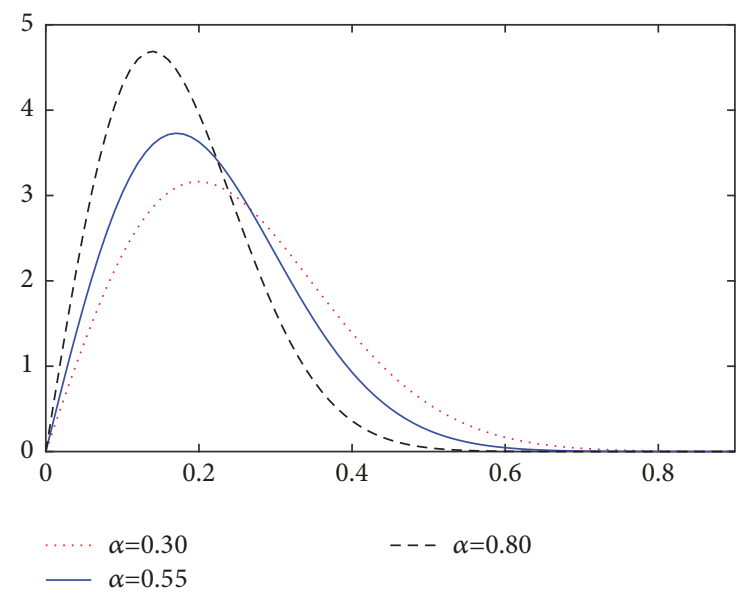

FIGURE 7: Effect of fractional order derivative on the probability function of amplitude.

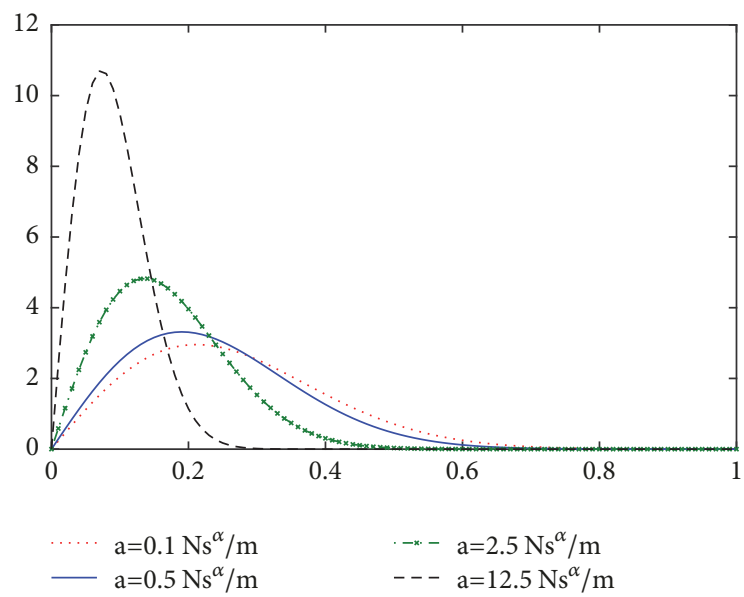

FIGURE 8: Effect of fractional damping on the probability function of amplitude.

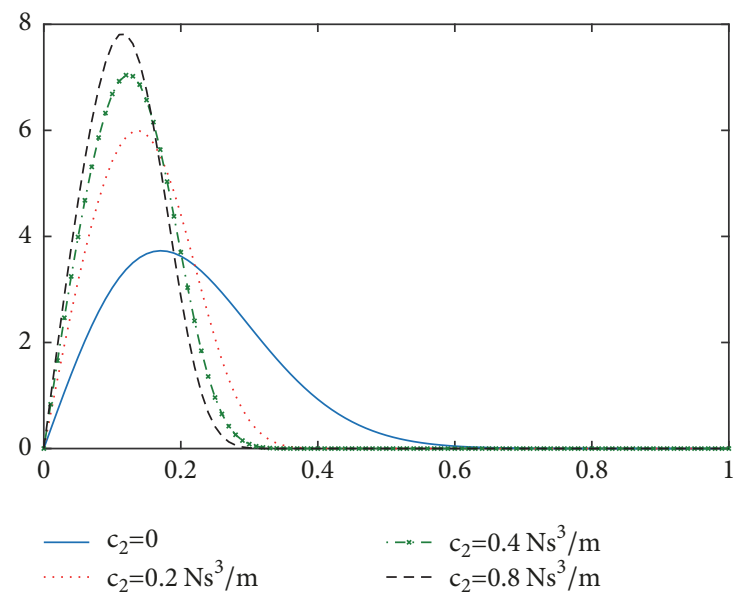

FIGURE 9: Effect of fractional damping on the probability function of amplitude.

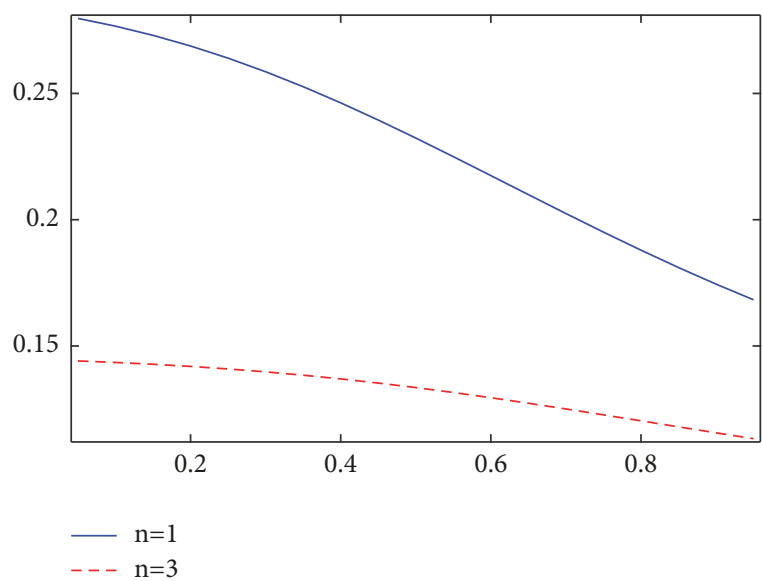

FIgURE 10: The mean amplitude E[A] as a function of $\alpha$.

nonlinear damping we can employ a material with a larger fractional order.

\section{Data Availability}

The data used to support the findings of this study are available from the corresponding author upon request.

\section{Conflicts of Interest}

The authors declare that they have no conflicts of interest.

\section{References}

[1] L. Debnath, "Recent applications of fractional calculus to science and engineering," International Journal of Mathematics and Mathematical Sciences, pp. 3413-3442, 2013.

[2] M. Dalir and M. Bashour, "Applications of fractional calculus," Applied Mathematical Sciences, vol. 4, pp. 1021-1032, 2010.

[3] Y. A. Rossikhin and M. V. Shitikova, "Application of fractional calculus for dynamic problems of solid mechanics: novel trends and recent results," Applied Mechanics Reviews, vol. 63, no. 1, Article ID 010801, 2010.

[4] F. C. Meral, T. J. Royston, and R. Magin, "Fractional calculus in viscoelasticity: an experimental study," Communications in Nonlinear Science and Numerical Simulation, vol. 15, no. 4, pp. 939-945, 2010

[5] D. Younesian, Z. Saadatnia, and H. Askari, "Analytical solutions for free oscillations of beams on nonlinear elastic foundations using the variational iteration method," Journal of Theoretical and Applied Mechanics, vol. 50, no. 2, pp. 639-652, 2012.

[6] Z. Xu and Y. K. Cheung, "Averaging method using generalized harmonic functions for strongly non-linear oscillators," Journal of Sound and Vibration, vol. 174, no. 4, pp. 563-576, 1994.

[7] A. Lal and B. N. Singh, "Stochastic nonlinear free vibration of laminated composite plates resting on elastic foundation in thermal environments," Computational Mechanics, vol. 44, no. 1, pp. 15-29, 2009.

[8] Z. L. Huang, W. Q. Zhu, and Y. Suzuki, "Stochastic averaging of strongly non-linear oscillators under combined harmonic and white-noise excitations," Journal of Sound and Vibration, vol. 238, no. 2, pp. 233-256, 2000. 
[9] W. Q. Zhu, Z. L. Huang, and Y. Suzuki, "Response and stability of strongly non-linear oscillators under wide-band random excitation," International Journal of Non-Linear Mechanics, vol. 36, no. 8, pp. 1235-1250, 2001.

[10] G.-G. Li, Z. Zheng-you, and C. Chang-jun, "Dynamical stability of viscoelastic column with fractional derivative constitutive relation," Applied Mathematics and Mechanics-English Edition, vol. 22, no. 3, pp. 294-303, 2001.

[11] P. D. Spanos and B. A. Zeldin, "Random vibration of systems with frequency-dependent parameters or fractional derivatives," Journal of Engineering Mechanics, vol. 123, no. 3, pp. $290-$ 292, 1997.

[12] P. D. Spanos and G. Malara, "Nonlinear Random Vibrations of Beams with Fractional Derivative Elements," Journal of Engineering Mechanics, vol. 140, no. 9, p. 04014069, 2014.

[13] P. D. Spanos and G. I. Evangelatos, "Response of a non-linear system with restoring forces governed by fractional derivativesTime domain simulation and statistical linearization solution," Soil Dynamics and Earthquake Engineering, vol. 30, no. 9, pp. 811-821, 2010.

[14] P. Wahi and A. Chatterjee, "Averaging oscillations with small fractional damping and delayed terms," Nonlinear Dynamics, vol. 38, no. 1-4, pp. 3-22, 2004.

[15] Y. Yang and W. Xu, "Stochastic analysis of monostable vibration energy harvesters with fractional derivative damping under Gaussian white noise excitation," Nonlinear Dynamics.

[16] Z. L. Huang and X. L. Jin, "Response and stability of a SDOF strongly nonlinear stochastic system with light damping modeled by a fractional derivative," Journal of Sound and Vibration, vol. 319, no. 3-5, pp. 1121-1135, 2009.

[17] L. C. Chen, Q. Q. Zhuang, and W. Q. Zhu, "Response of SDOF nonlinear oscillators with lightly fractional derivative damping under real noise excitations," The European Physical Journal Special Topics, vol. 193, no. 1, pp. 81-92, 2011.

[18] L. Chen and W. Zhu, "First passage failure of SDOF nonlinear oscillator with lightly fractional derivative damping under real noise excitations," Probabilistic Engineering Mechanics, vol. 26, no. 2, pp. 208-214, 2011.

[19] L. Chen, Q. Lou, Z. Li, and W. Zhu, "Stochastic stability of the harmonically and randomly excited Duffing oscillator with damping modeled by a fractional derivative," Science China Physics, Mechanics \& Astronomy, vol. 55, no. 12, pp. 2284-2289, 2012.

[20] L. Chen, H. Li, Z. Li, and W. Zhu, "Asymptotic stability with probability one of MDOF nonlinear oscillators with fractional derivative damping," Science China Physics, Mechanics \& Astronomy, vol. 56, no. 11, pp. 2200-2207, 2013.

[21] L. Chen, W. Wang, Z. Li, and W. Zhu, "Stationary response of Duffing oscillator with hardening stiffness and fractional derivative," International Journal of Non-Linear Mechanics, vol. 48, pp. 44-50, 2013.

[22] Y. Yang, W. Xu, W. Jia, and Q. Han, "Stationary response of nonlinear system with Caputo-type fractional derivative damping under Gaussian white noise excitation," Nonlinear Dynamics, vol. 79, pp. 139-146, 2014.

[23] Y. G. Yang, W. Xu, X. D. Gu, and Y. H. Sun, "Stochastic response of a class of self-excited systems with Caputo-type fractional derivative driven by Gaussian white noise," Chaos, Solitons \& Fractals, vol. 77, pp. 190-204, 2015.

[24] G. Failla and A. Pirrotta, "On the stochastic response of a fractionally-damped Duffing oscillator," Communications in
Nonlinear Science and Numerical Simulation, vol. 17, no. 12, pp. 5131-5142, 2012.

[25] Y. Sun, L. Hong, J. Jiang, and Z. Li, "Estimation of critical conditions for noise-induced bifurcation in nonautonomous nonlinear systems by stochastic sensitivity function," International Journal of Bifurcation and Chaos, vol. 26, no. 11, 1650184, 12 pages, 2016.

[26] L. Chen, T. Zhao, W. Li, and J. Zhao, "Bifurcation control of bounded noise excited Duffing oscillator by a weakly fractionalorder $P I_{\lambda} D_{\mu}$ feedback controller," Nonlinear Dynamics, vol. 83, no. 1-2, pp. 529-539, 2016.

[27] L. Chen and J.-Q. Sun, "The closed-form solution of the reduced Fokker-Planck-Kolmogorov equation for nonlinear systems," Communications in Nonlinear Science and Numerical Simulation, vol. 41, pp. 1-10, 2016.

[28] D. Yurchenko, A. Burlon, M. Di Paola, G. Failla, and A. Pirrotta, "Approximate Analytical Mean-Square Response of an Impacting Stochastic System Oscillator With Fractional Damping," ASCE-ASME J. Risk and Uncert. in Engrg. Sys., Part B: Mech. Engrg., vol. 3, no. 3, p. 030903, 2017.

[29] Y. Yang, W. Xu, and G. Yang, "Bifurcation analysis of a noisy vibro-impact oscillator with two kinds of fractional derivative elements," Chaos: An Interdisciplinary Journal of Nonlinear Science, vol. 28, no. 4, 043106, 8 pages, 2018.

[30] H. S. Shen, J. Yang, and L. Zhang, "Free and forced vibration of Reissner-Mindlin plates with free edges resting on elastic foundations," Journal of Sound and Vibration, vol. 244, no. 2, pp. 299-320, 2001.

[31] X.-L. Huang and J.-J. Zheng, "Nonlinear vibration and dynamic response of simply supported shear deformable laminated plates on elastic foundations," Engineering Structures, vol. 25, no. 8, pp. 1107-1119, 2003.

[32] T. G. Kostantakopoulos and G. T. Michaltsos, "Modeling and analysis of a plate on elastic foundation subjected to landing airplanes' forces," International Journal of Structural Stability and Dynamics, vol. 10, no. 1, pp. 37-54, 2010.

[33] H. Dang-Trung, H. Luong-Van, T. Nguyen-Thoi, and K. K. Ang, "Analyses of stiffened plates resting on viscoelastic foundation subjected to a moving load by a cell-based smoothed triangular plate element," International Journal of Structural Stability and Dynamics, vol. 17, no. 1, 1750011, 28 pages, 2017.

[34] W. Cai, W. Chen, and W. Xu, "Fractional modeling of Pasternaktype viscoelastic foundation," Mechanics of Time-Dependent Materials, vol. 21, no. 1, pp. 119-131, 2017.

[35] S. Y. Reutskiy, "A new numerical method for solving high-order fractional eigenvalue problems," Journal of Computational and Applied Mathematics, vol. 317, pp. 603-623, 2017.

[36] S. Y. Reutskiy, "A novel method for solving second order fractional eigenvalue problems," Journal of Computational and Applied Mathematics, vol. 306, pp. 133-153, 2016.

[37] G. Malara and P. D. Spanos, "Nonlinear random vibrations of plates endowed with fractional derivative elements," Probabilistic Engineering Mechanics, vol. 54, pp. 2-8, 2018.

[38] D. Younesian, H. Askari, Z. Saadatnia, and M. KalamiYazdi, "Frequency analysis of strongly nonlinear generalized Duffing oscillators using HE's frequency-amplitude formulation and HE's energy balance method," Computers \& Mathematics with Applications, vol. 59, no. 9, pp. 3222-3228, 2010.

[39] S. Farhangdoust, D. Younesian, and E. Esmailzadeh, "Interaction of Higher Modes in Nonlinear Free Vibration of Stiffened Rectangular Plates," in Proceedings of the ASME 2017 International Design Engineering Technical Conferences and Computers 
and Information in Engineering Conference, p. V008T12A043, Cleveland, Ohio, USA.

[40] Haiwu Rong and Xiangdong Wang, "Subharmonic Response of Linear Vibroimpact System with Fractional Derivative Damping to a Randomly Disrobed Periodic Excitation," Mathematical Problems in Engineering, vol. 2015, pp. 1-10, 2015.

[41] Ying Li and Ye Tang, "Analytical Analysis on Nonlinear Parametric Vibration of an Axially Moving String with Fractional Viscoelastic Damping," Mathematical Problems in Engineering, vol. 2017, pp. 1-9, 2017. 


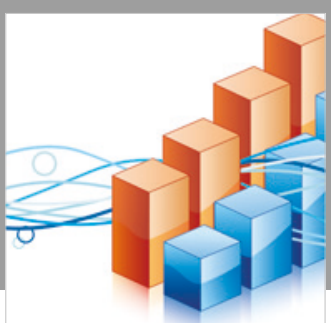

Advances in

Operations Research

\section{-n-m}
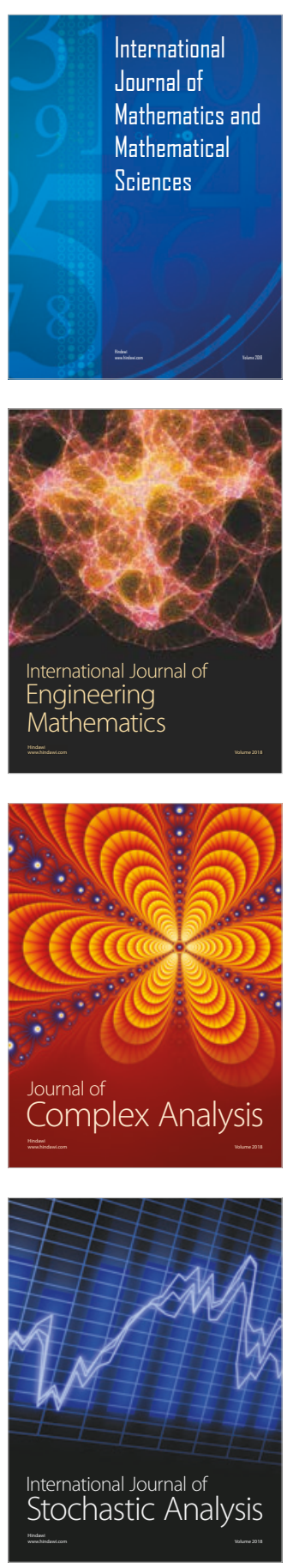
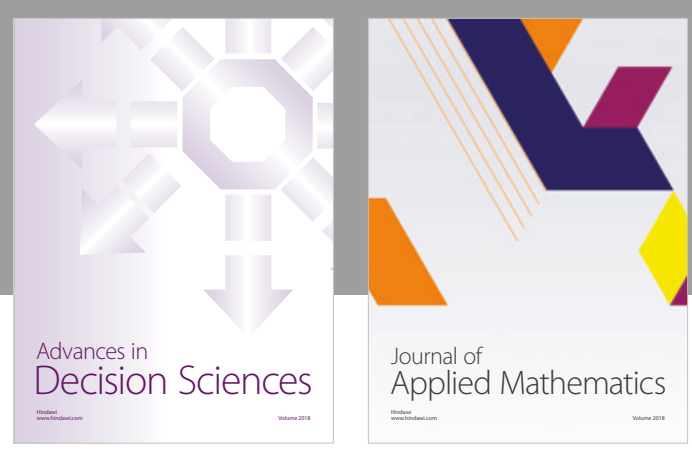

Journal of

Applied Mathematics
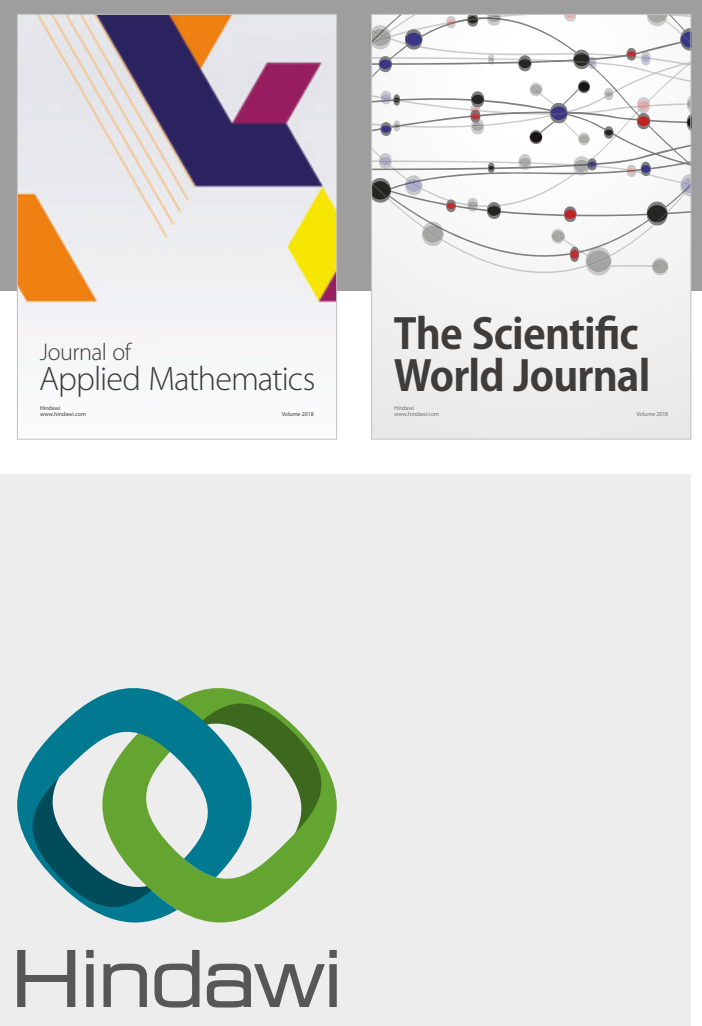

Submit your manuscripts at

www.hindawi.com

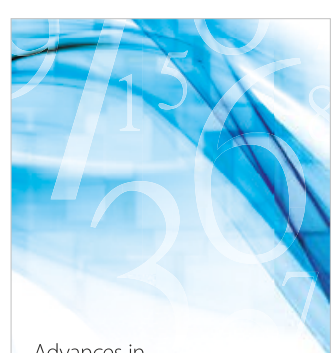

Advances in
Numerical Analysis
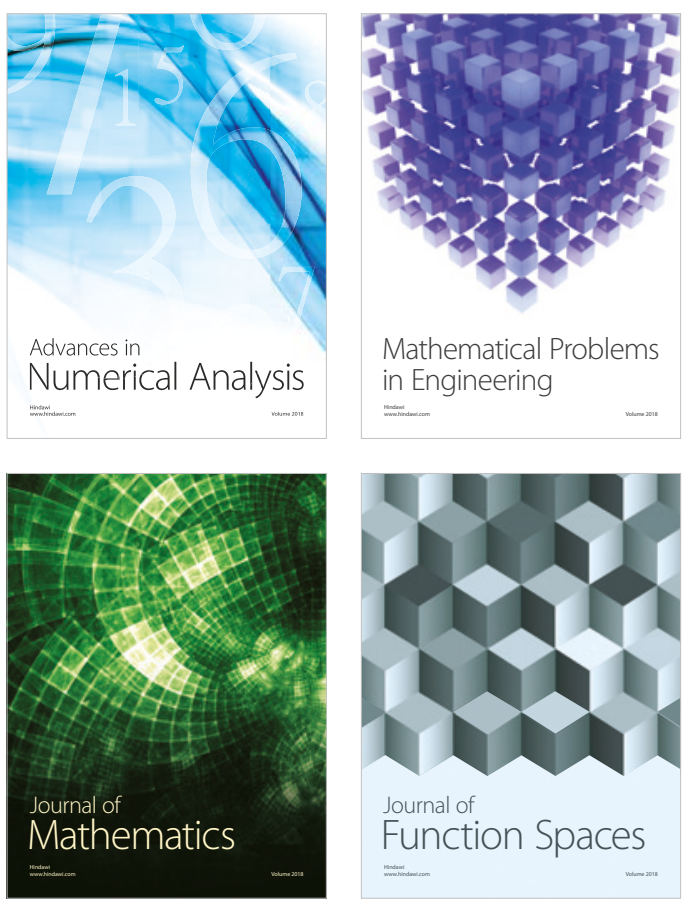

Mathematical Problems in Engineering

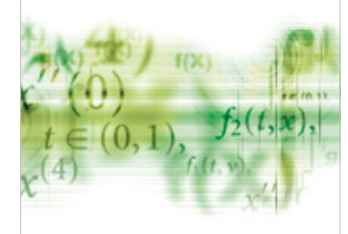

International Journal of

Differential Equations

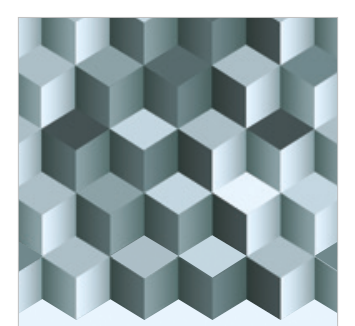

Journal of

Function Spaces

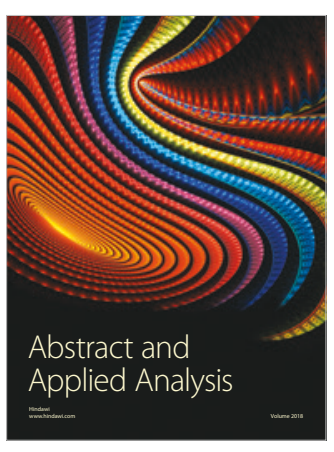

The Scientific

World Journal

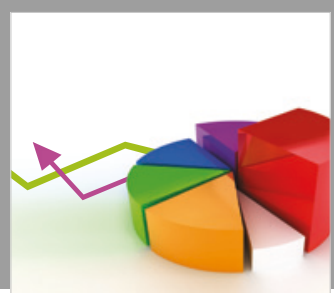

Journal of

Probability and Statistics
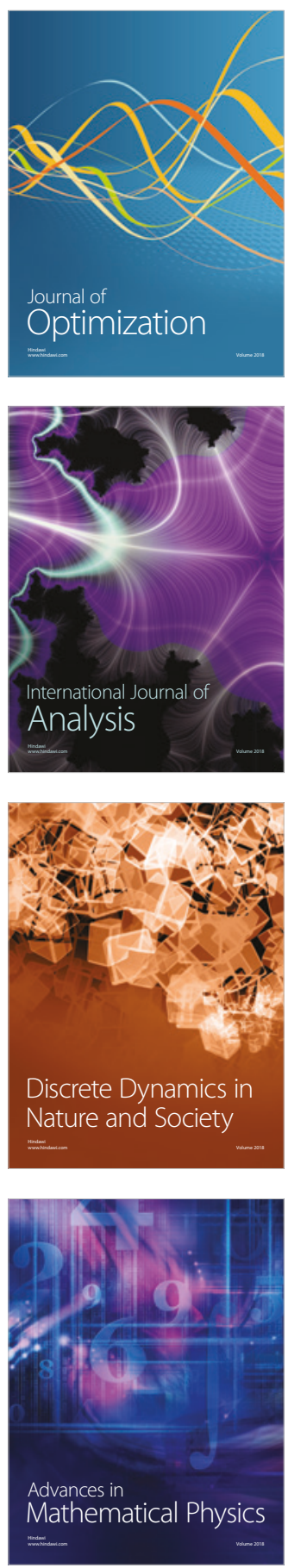